\title{
An Elliptically Flared Waveguide Horn
}

\author{
J.A.G. Malherbe and Y. Katcodia \\ Department of Electrical, Electronic and Computer Engineering \\ University of Pretoria \\ Pretoria 0002, South Africa \\ jagm@up.ac.za
}

ABSTRACT - An X-band waveguide horn of which the sides flare by an elliptic function is described. Measurements performed show that the structure exhibits an absence of side lobes in the conventional sense, and that the radiation pattern varies very little over the entire X-band. It has almost equal beamwidth in the principal planes, and the input return loss is extremely low.

Key Words: Waveguide horn, elliptic profile

\section{INTRODUCTION}

Conventional pyramidal waveguide horns find very wide application both as primary antennas and as feeds for reflector systems. They exhibit useful gain and radiation properties, although their radiation patterns tend to vary strongly with frequency. In an effort to improve some of the properties of pyramidal horns, authors have described modifications such as to the throat of a sectoral horn [1], and the introduction of cylindrical edges to reduce diffraction and match the aperture [2]. The profiling of corrugated horns to achieve Gaussian beams, as well as other profile options to achieve this purpose have been described [3] - [8].

The horn antenna described in [9] was designed as an impedance match between an X-band waveguide and free space, and it was seen that extremely low values of reflection coefficient were obtained. At the same time the radiation pattern exhibited a lack of side lobes due to the spherical wave front generated by the antenna. Lately, it has been demonstrated that TEM horns with an elliptic flare profile are singularly well matched, and exhibit almost frequency independent properties [10], [11]. In this paper, the principle of an elliptic flare is extended to an $\mathrm{X}$-band waveguide horn in which both sides flare to form elliptic profiles.

\section{HORN CONSTRUCTION}

The horn flares from the dimensions of an X-band waveguide to an aperture of dimensions $150 x 150 \mathrm{~mm}$, over a total length of $200 \mathrm{~mm}$, as shown in Fig. 1. For the flare on the broad side of the waveguide, Fig. 1(a), the ellipse major axis is $a=$ $400 \mathrm{~mm}$, while the minor axis is $b=150-22.86=127.14 \mathrm{~mm}$. The flare on the narrow side of the waveguide has the same major axis, but a minor axis of $b=$ $139.84 \mathrm{~mm}$, so that the aperture has dimensions of $150 \mathrm{x} 150 \mathrm{~mm}$.

The horn is constructed of $0.5 \mathrm{~mm}$ plate and soldered to a standard X-band waveguide flange.

Prior to manufacture, a numerical model was created with the commercial software FEKO [12], and reflection coefficient, gain and radiation patterns were evaluated. 

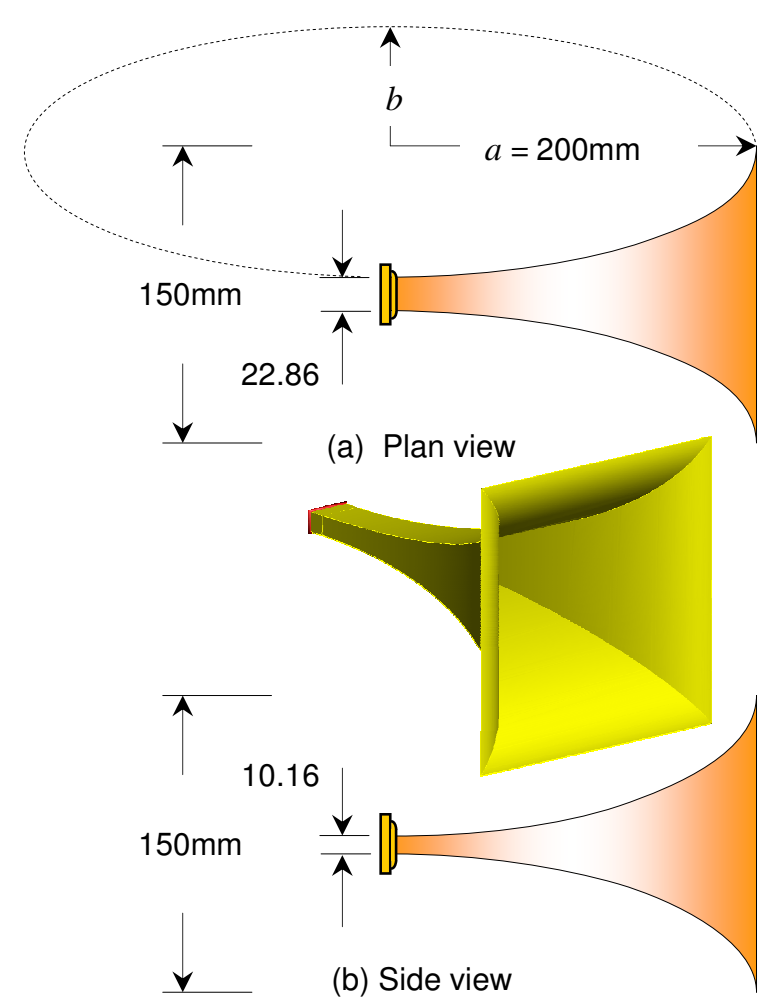

Fig. 1. Plan (a) and side (b) views of the elliptic horn

\section{MEASUREMENTS}

Fig. 2 shows the gain and the return loss of the horn over the standard X-band frequency range. Calculated and measured gain are in good agreement; measured gain varies between 16.5 and $19 \mathrm{~dB}$. The antenna is extremely well matched, and while the calculated match is substantially better, the measured return loss is of the same order as that of the best matched load. Small errors in the construction of the horn, especially in the throat region, will cause a deterioration in the measured return loss.

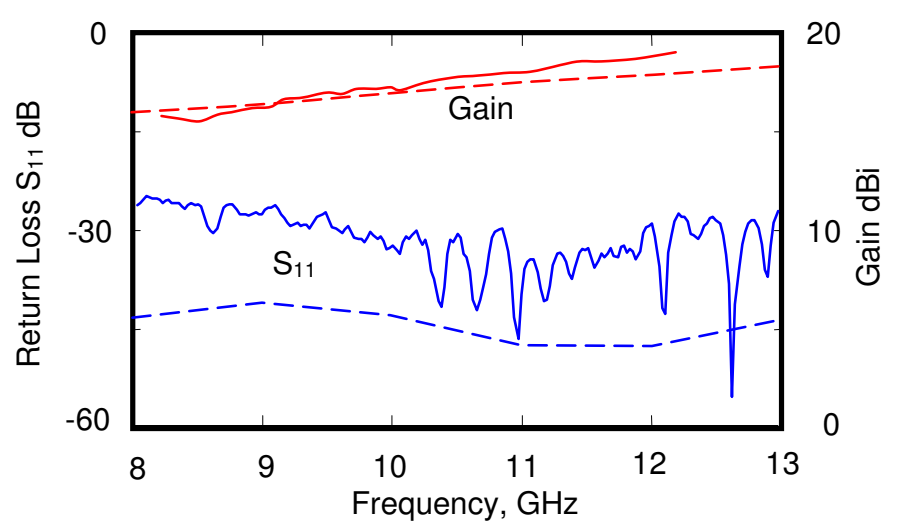

Fig. 2. Gain and return loss response vs frequency measured - - - calculated

Fig 3(a) shows the measured E-plane radiation patterns for frequencies from 8 to $13 \mathrm{GHz}$ in $1 \mathrm{GHz}$ steps, while the corresponding patterns for the H-plane are shown in Fig. 3(b). The peak regions of the main lobe of both E- and H-plane are shown expanded in Fig. 4. The beamwidths over the entire frequency range of 8 to $13 \mathrm{GHz}$ are extremely constant; the $-3 \mathrm{~dB}$ beamwidth in the $\mathrm{H}$-plane amounts to $25.5^{\circ} \pm 3.5^{\circ}$, 
while the E-plane beamwidth is $27^{\circ} \pm 5^{\circ}$. At $-10 \mathrm{~dB}$ the beamwidth in the H-plane is $48.5^{\circ} \pm 6.5^{\circ}$ and for the E-plane $58.5^{\circ} \pm 6.5$.

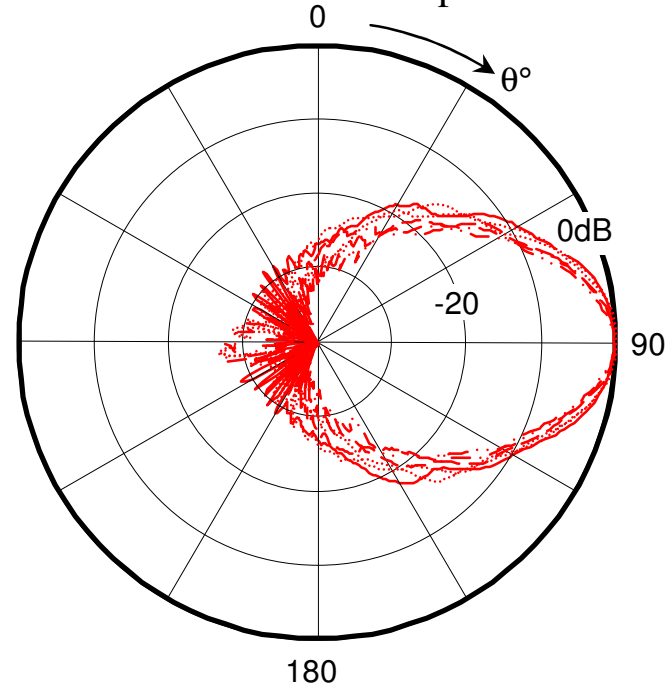

(a)

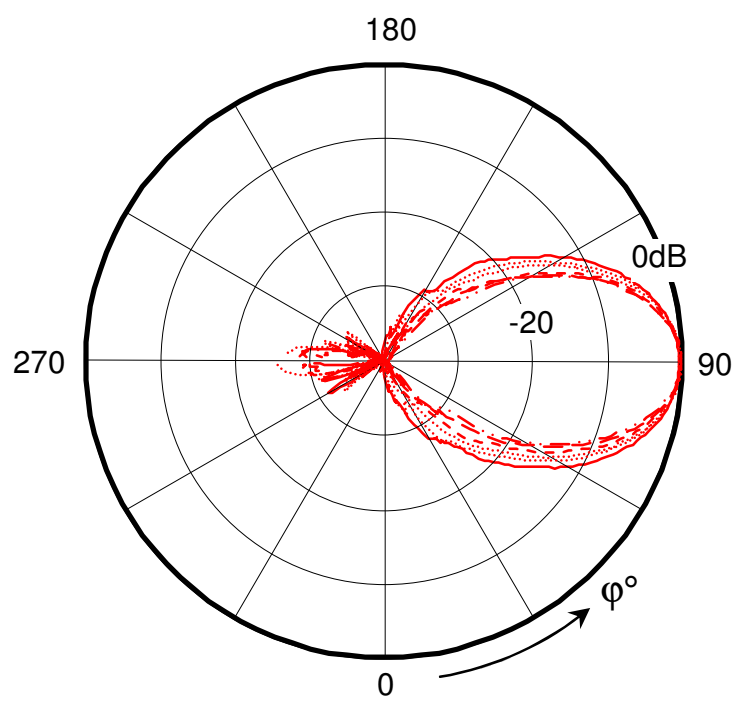

(b)

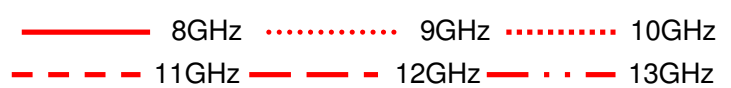

Fig. 5 compares the calculated and measured radiation patterns for both principal planes at $10 \mathrm{GHz}$; for all practical purposes calculated and measured patterns are identical.

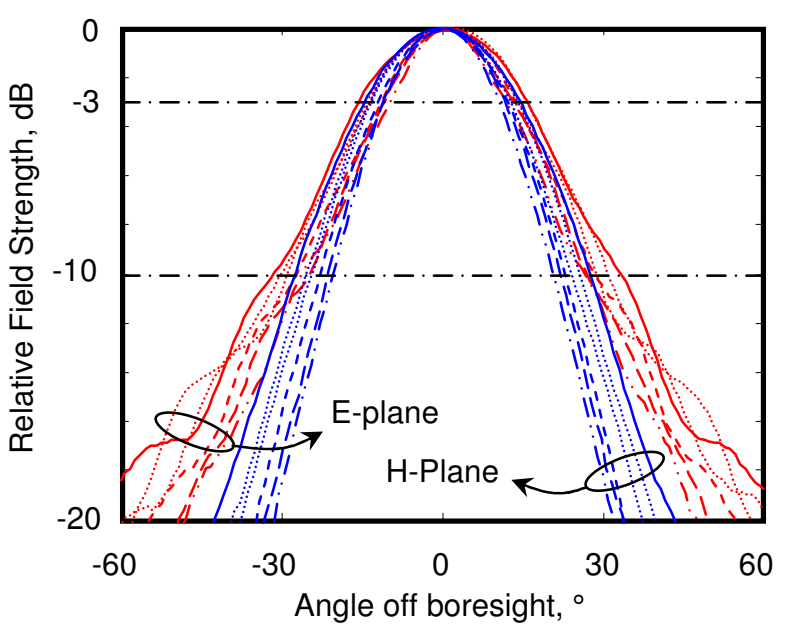

Fig. 4. Expanded radiation pattern, both E- and H-Planes, 8-13GHz
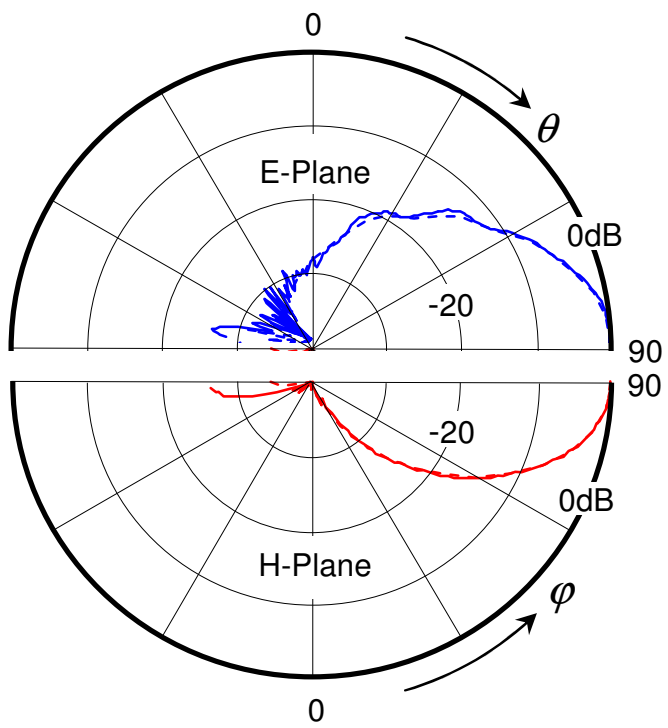

Fig. 5. Comparison of measured and calculated radiation patterns, E-Plane (upper) and $\mathrm{H}$-plane (lower) at $10 \mathrm{GHz}$. - - - - calculated measured

\section{CONCLUSION}

The $\mathrm{TE}_{10}$ mode that propagates down the horn has nulls in the H-plane of the electric field parallel to the sides of the horn, causing the H-plane pattern to be slightly sharper than the E-plane pattern at angles well rmoved from boresight. Because of the finite values of the E field normal to the horn upper plates, the scattered back lobe is slightly larger in the E-plane. 
The sidelobes that would normally be associated with a pyramidal horn, in the case of this horn, degenerate almost completely due to the wide flare of the horn and the launching of a spherical wave front.

The horn is extremely well matched to the X-band waveguide for which it was designed, and the gain varies smoothly with frequency. The E- and H-plane radiation patterns are very nearly the same, and vary very little over frequency, making the elliptically flared horn well suited to feeding a parabolic dish.

\section{REFERENCES}

[1] H. Henke, "Influence of the Wall Geometry on Reflection and Transmission in an EPlane Sectoral Horn", IEEE Trans. Antennas Propagat., Vo. 25, No. 4, pp. 556-560, Jul. 1977.

[2] W.D. Burnside and C.W. Chuang, "An Aperture-Matched Horn Design”, IEEE Trans. Antennas Propagat., Vol. 30, No. 4, pp. 790-796, July 1982.

[3] S.F. Mahmoud, "Mode conversion on profiled corrugated conical horns", IEE Proc., Vol. 130, Pt. H, No. 6, pp. 415-419, Oct 1983.

[4] A.D. Olver and J. Xiang, "Design of Profiled Corrugated Horns", IEEE Trans Antennas Propagat., Vol. 36, No. 7, pp. 936-940, Jul. 1988.

[5] B. Philips and A.D. Olver, "Design and performance of profiled dielectric loaded horns", IEE Proc. Microw. Antennas Propagat., Vol. 141, No. 5, pp. 337-341, Oct 1994.

[6] R. Gonzalo, J. Teniente, and C del Río, "Improved Radiation Pattern Performance of Gaussian Profiled Horn Antennas", IEEE Trans. Antennas Propagat., Vol. 50, No. 11, pp. 1505-1513, Nov. 2002.

[7] C. Granet, "Profile options for feed horn design", 2000 Asia-Pacific Microwave Conf., Dec. 2000, pp. 1448-1451

[8] C. Granet, G.L. James, R Bolton, and G. Moorey, "A Smooth-Walled Spline-Profile Horn as an Alternative to the Corrugated Horn for Wide Band Millimeter-Wave Applications", IEEE Trans. Antennas Propagat., Vol. 52, No. 3, pp. 848-854, Mar. 2004.

[9] J.A.G. Malherbe, "A Compact Horn Antenna with Low Return Loss," 1986 IEEE AP-S Int. Antenna. Symp. Dig. Vol.2, pp.743-746, Philadelphia, USA, June 1986.

[10] J.A.G. Malherbe, "Extreme Performance TEM Horn", Microwave Optical Tech. Lett., Vol. 50, No. 8, pp. 2121-2125, Aug. 2008.

[11] J.A.G. Malherbe, "Frequency-Independent Performance of Elliptic Profile TEM Horns", Microwave Optical Tech. Lett, Vol. 51, No. 3, pp. 607-612, March 2009.

[12] FEKO® EM Software \& Systems-S.A. (Pty) Ltd. http://www.feko.info/index.html. 